DIVISION OF THE HUMANITIES AND SOCIAL SCIENCES

CALIFORNIA INSTITUTE OF TECHNOLOGY

PASADENA, CALIFORNIA 91125

PRINCIPLES OF NETWORK DEVELOPMENT AND EVOLUTION: AN EXPERIMENTAL STUDY

Steven Callander

Northwestern University

Charles R. Plott

California Institute of Technology

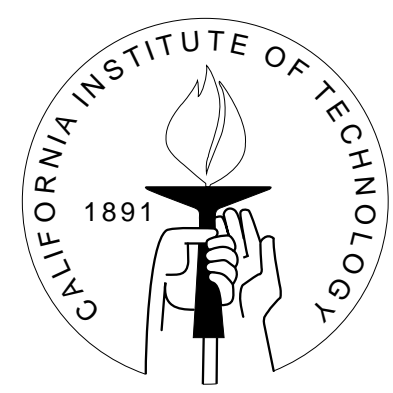

SOCIAL SCIENCE WORKING PAPER 1156R

July 2002

December 2003 


\title{
Principles of Network Development and Evolution: An Experimental Study*
}

\author{
Steven Callander ${ }^{\dagger}$ \\ Charles R. Plott $\ddagger$ \\ Northwestern University California Institute of Technology \\ July 2002 \\ Revised: December 14, 2003
}

\begin{abstract}
This paper reports on an experimental investigation of the evolution of networks and the individual decision making processes that guide it. Since there is no history of experimental work on network formation, part of the paper is devoted to the formulation of problems that can be examined experimentally. The results are that networks, composed of decentralized decision makers, are capable of overcoming complex coordination and learning problems and converge to stationary configurations. While stationarity is frequently observed, such an achievement is not guaranteed and when it doesn't occur significant and persistent inefficiencies can result. The models of equilibration based on the principle of Nash equilibrium are more reliable than models based on the alternative principles of efficiency seeking or focalness of the network configuration. However, individual decision making within networks is not in accordance with the simple decision rule of Nash best response. Instead we observe complicated strategies that appear to trade short term profits in order to signal to, and teach, other agents the strategies required for long term profit maximization.
\end{abstract}

*The financial support of the Laboratory of Experimental Economics and Political Science at Caltech is gratefully acknowledged.

$\dagger$ Assistant Professor of Managerial Economics and Decision Sciences, MEDS department, Kellogg School of Management, Northwestern University.

${ }^{\ddagger}$ Professor of Economics and Political Science, Division of the Humanities and Social Sciences, California Institute of Technology. 


\section{Introduction}

A central challenge to all public oriented research is the isolation of principles that govern the self-organizing and evolutionary properties of institutions. For that reason networks and network formation are natural phenomena to study. Network structures, both formal and informal, are often the means by which information, goods, or services flow through the economy and society. Increasingly over recent years, researchers have used network concepts to model and understand a broad array of important environments. Examples include (but are not limited to) labor market participation, ${ }^{1}$ industry structure, ${ }^{2}$ the internal organization of firms, ${ }^{3}$ and sociological interactions covering such broad phenomena as social norms, peer pressure and the attainment of status. ${ }^{4}$

Underlying networks in all manifestations are important public economic questions of efficiency and distribution. Economic networks as well as networks that facilitate the information flow of broad social and political activities, often develop and evolve in a decentralized, self-organizing manner. However, the complexities of such processes are underscored by the reality that important networks are also developed within the framework of a regulated monopoly, suggesting a failure of decentralized decisions, perhaps due to network features of public goods, multilateral negotiation and coordination, in which decentralized decisions are thought to work inefficiently. Thus, natural questions to pose focus on the principles that might be at work, guiding the unregulated development and evolution of networks. Two key questions are preeminent: (1) What principles underlie network development and evolution? and (2) How can the principles be understood in the context of individual decisions and behavior? Hopefully, the answers will be useful for the design of policies that help facilitate

\footnotetext{
${ }^{1}$ This is perhaps the broadest application of networks within economics; see Montgomery (1991) for a discussion and references. The focus of this literature is on the flow of information about job opportunities through social and other networks. In a recent application, CalvoArmengol and Jackson (2003) show how particular network structures may preclude equality of opportunity in the labor market across groups.

${ }^{2}$ An excellent example is Kranton and Minehart (2001).

${ }^{3}$ See, for example, Keren and Levhari (1983).

${ }^{4}$ There exists a large and broad literature in sociology addressing these issues (see Wellman and Berkowitz (1988)). In an economic context many of the issues are dealt with, either explicitly or implicitly, in the special issue of the Journal of Public Economics (1998, Vol. 4) dealing with status. For example, Neumark and Postlewaite (1998) show that family networks and relative income play an important role in decisions of married women to reenter the workforce.
} 
efficient network formation and functioning.

At an abstract level the questions provide a natural arena for the machinery of game theory, and in recent years a burgeoning literature has arisen (the seminal contribution is Jackson and Wolinsky (1996); see Jackson (2003) for a recent survey). The game theoretic literature has provided enormous insight into networks, most prominently exposing a critical tension in many network environments between efficiency and individual incentives. The implication of this insight is that "stable" network configurations (those that persist through time) may not be efficient.

Questions of efficiency may, however, be moot if network structures do not evolve towards some predictable stationary states. An influential strand of the game theoretic literature, pioneered by Bala and Goyal (2000), has explored network dynamics and provided a remarkable result. In a decentralized network environment with self-interested and myopic decision makers, Bala and Goyal (2000) developed a model of how network structures can rapidly evolve in a predictable manner to stationary configurations. The work serves to not only confirm the theoretical relevance of networks, but powerfully demonstrate that, despite overwhelming complexity, the individual decision maker is a tractable unit of analysis.

Behind the mathematical elegance of the models, however, particular behavioral principles and solution concepts underlie all game theoretic analyses, both static and dynamic. Moreover, these principles, while intuitively plausible, have often proved inadequate at describing behavior at the individual level in other environments (most notably with public goods). ${ }^{5}$ Therefore, at an applied level the key questions of individual behavior within networks and aggregate dynamics remain open.

Unfortunately, given the complexity, breadth and decentralized nature of naturally occurring networks, extracting evidence from field data on the reliability of the principles is inherently problematic. Consequently, we turn to laboratory experiments. To avoid the difficulties of field data we consider simple environments and small group interactions while carefully controlling individual incentives and the sequencing of network evolution.

Our primary results are as follows. Networks do spontaneously emerge and are capable of converging to configurations that remain stationary from round to

\footnotetext{
${ }^{5}$ As Ledyard (1995, p. 172) was left to conclude in a review of public goods experiments, "if these experiments are viewed solely as tests of game theory, that theory has failed." As will become clear, this failure is particularly relevant as networks share several common features with public goods.
} 
round. While convergence to stationary networks does not always occur, when it does occur the network is of a predictable form. Significantly, the dynamics of network formation does not reliably exhibit monotonically increasing efficiency. Approximately speaking, at certain critical points in network dynamics the coordination, bargaining and free rider aspects of individual decision making become aligned and stationarity is achieved. At these points it appears that all decision makers become aware of which network is best for them, and are aware that other agents are aware of this, and so on ad infinitum. In non-convergent networks this coordination of beliefs appears to fail and significant inefficiency results.

We discover the principle behind convergence and network dynamics to be Nash-like (although not necessarily strict Nash), as opposed to efficiency or focalness. These findings allow us to answer a fundamental question: game theory and its concepts of equilibria are appropriate to be applied to the network problem (and thus the models of Jackson and Wolinsky, and others are not idle mathematical speculations).

At the outset it is important to emphasize that the experiments reported here are "exploratory." No previous network experiments exist on which to build. The number of variables is staggering and there is no obviously best configuration with which to start. The elementary state of the theory together with the number of variables suggest that a "measurement" approach to experiments will not work. It makes little sense to measure the effects of some single, particular variable when neither the theory nor the importance of the variables are well established. So, as is the case with exploratory methodology, part of the problem addressed in the study involves questions about where to start and in what directions one might push. The overall development of the paper is designed to explain the considerations that were made in the approach so the study can be used as a benchmark for others who feel that alternative directions might be more productive.

The paper is developed as follows. Section 2 presents a brief introduction to the formal concept of networks. It also contains a summary of the variables that have been used in the literature and the theoretical models of networks to be used in the experiments. Section 3 presents the overall experimental design and the features of both Series 1 and 2 of experiments. Section 4 presents the results for both series of experiments and section 5 concludes the paper. 


\section{Experimental Setting and Network Models}

As mentioned in the introduction, the experimental design resides in the domain of "exploratory" methodology. The approach is dictated by both the lack of previous experiments together with the abundance of variables and a corresponding incompleteness of theory. The approach is to explore proposed general principles that the literature suggests might govern network development and evolution.

Naturally occurring networks take place in a variety of institutional and informational environments. However, many have the property of repeated interactions among a set of agents (and not as one-shot interactions). As such, our experiments involved repeated decisions by a fixed set of agents. This structure gives rise to three basic questions: (1) do networks converge to steady state outcomes and, if so, what are the properties of the state? (2) What principles drive the evolution of networks? (3) How is the process influenced by the institutional environment? In the remainder of this section candidate models are outlined as potential answers to the first two questions. Answers to the third question remain open but answers to the first two are suggestive.

\subsection{Network Environments}

A network is a set of connections that join distinct nodes together. We study networks in which each node is a separate decision making agent. Each agent unilaterally chooses the links they form between themselves and other agents. ${ }^{6}$ At each node exists a "piece" of information (this can be interpreted alternatively as a good or service, social pressure, norms of behavior etc.) that has the capacity to flow through the network. All information that exists at the node to which a connection is being made is passed to the node that initiated the connection. The benefit is received by each node through which the information passes, including the node of origin (the benefit can be received only once). The value of information to an agent is independent of the number of links that it passes through before reaching an agent, and so we say information flow is without "decay." Links are reliable (i.e., never fail) and are assumed to

\footnotetext{
${ }^{6}$ There are several other literatures that proceed under the banner of "networks" that differ significantly and will not be considered here. These include the literature on airline networks (see Hendricks, Piccione, and Tan (1999)) and network externalities.
} 
be one-way and are paid for by the connecting agent, who receives the benefit. ${ }^{7}$ Each agent is free to connect to any other agent, or combination of agents, that he chooses. The timing and knowledge on which agent decisions can be based are discussed later in this and the succeeding section.

An example of a network with six agents is depicted in Figure 1. Our experiments all involved six agents, a size reflecting a trade-off between capturing network complexity while maintaining manageability.

** Figure 1 about here.

In the network of Figure 1 each agent chose to implement only one link. The direction of the arrow points to the agent who constructed the link and receives the information flow. In this particular network each agent receives every available piece of information because the sequence of links traces continuously through every node in the network.

Agents within the network receive continuously updated information about the structure of the network in which they are operating. Thus, from a modeling perspective a natural beginning would be a model in which the structure of the network is common knowledge. Of course, the physical realities of presenting such information to subjects must be acknowledged. Exactly how that can be done and how the information must be organized will be discussed in the experimental procedures section. Nevertheless, we proceed on the assumption that each agent has full information about the size and composition of the network, as well as the links selected by other agents. There is no communication of any kind between the agents other than through their link choice.

\subsection{Network Structures and Models of Network Forma- tion}

In this section three broad principles of network formation are introduced: Nash equilibrium, efficiency and focalness. These principles provide predictions about network configurations that may prove stationary as well as the

\footnotetext{
${ }^{7}$ Bala and Goyal (2000a) explore the implications if links are not reliable. An alternative specification may assume links are two-way (and so information flows both ways). Two-way links adds the issue of who will pay for the link as it benefits both connected agents. Bala and Goyal (2000) assume the instigator of the link pays, which adds a further coordination problem among agents (each will wait for the other to connect). To avoid this further complication we assume links are one-way.
} 
"directions" network evolutions might take. In this sense the principles can serve as both models of stationary configurations as well as individual action and movements of configurations.

\subsubsection{Principles}

Nash equilibrium is standard from the theory of games. A configuration is a Nash equilibrium if given what other agents are doing, no individual can improve personal gains by some unilateral change of action. We will consider both strict and weak definitions of Nash equilibrium. It is important to note that we employ the static definition of Nash equilibrium (i.e., the equilibrium of a one-shot game) although the environments we consider are dynamic. Strategic incentives within dynamic networks are little understood and a characterization of equilibrium is not available. ${ }^{8}$

Efficiency refers to the proportion of gains received by all agents relative to all potential gains, without regard to the individuals that receive the gains. If gains are the maximum possible then the system is at $100 \%$ efficiency. Such a calculation reflects both the distribution of information around the network and the cost of the formation of the network.

Focalness, is not usually considered in formal models since in the world of abstract reasoning there is not typically a commonly held sense of position. In contrast, subjects in the experiments share a geographical space that may provide a coordinating device for the agents, as originally discussed by Schelling (1960). The application of focalness for purposes of this paper reflect the positions that subjects might have been placed in the room, the positions in which data were put on the chalkboard or the positions in which individuals appeared in network representations on screens.

\subsubsection{Parameters and Predictions}

The three principles described above, for different parameter values, may lead to identical or divergent predictions. Table 1 describes four sets of network parameters in which the predictions of these principles converge and diverge. The Nash equilibrium, efficient, and focal networks for these different parameter values are described in Table 2. These parameter values (and their divergent

\footnotetext{
${ }^{8}$ Although, given the multitude of one-shot Nash equilibria that often exist, a folk theorem result most likely holds.
} 
predictions) will be exploited in our experimental design to establish a convergence result and then to distinguish between the predictions.

** Tables 1 and 2 about here.

Parameter sets 1 and 2 involve symmetric costs and benefits and lead to identical predictions. These parameters fit the model of Bala and Goyal (2000) who showed that the "wheel network" is uniquely efficient and strict Nash. As the name suggests, a wheel network requires each agent to connect only one link from another agent such that these links form one long chain. Although, this chain need not appear as a wheel when depicted graphically. Examples of wheel networks are given in Figure 2 below and Figure 1 earlier. This architecture $^{9}$ is efficient as all agents receive maximum value for the cost of only one link. While the two configurations in Figures 1 and 2 are equivalent with respect to efficiency and Nash equilibrium, focalness draws a distinction between them. Agents in our experiments are seated as depicted in the figures and are assigned consecutive numbers as indicated. Therefore, we assume that the wheel in Figure 2 is focal (the counter-clockwise wheel), whereas the wheel in Figure 1 is not.

** Figure 2 about here.

It is important to note that even though the wheel network is the unique strict Nash equilibrium, there exists many weak Nash equilibria. Figure 3 provides an example with eight links.

\section{** Figure 3 about here.}

Parameter sets 1 and 2 were used in our experiments to give convergence "its best shot" and establish the capability for network equilibration. With equilibration established (at a focal wheel), we sought to distinguish between the three potential guiding principles. Parameter sets 3 and 4, which are outside the domain analyzed by Bala and Goyal (2000), distinguish between the principles by relaxing the symmetry and anonymity of link costs. ${ }^{10}$ Parameter set 3 imposes higher costs on links made between neighboring nodes. ${ }^{11}$ The

\footnotetext{
${ }^{9}$ Two networks have the same architecture, as defined by Bala and Goyal (2000, p. 1182), if one network can be obtained from the other by permuting the strategies of agents in the other network.

${ }^{10}$ Bala and Goyal (2000) do not asuume linearity of payoffs (as assumed in parameter sets 1 and 2) but they do impose symmetry and anonymity.

${ }^{11}$ Neighbors are defined as geographically adjacent agents. For example, the neighbors of agent 6 are 5 and 1.
} 
asymmetry immediately implies that the focal wheels, which rely exclusively on neighborly links, are no longer efficient. In this environment the efficient configurations are wheels in which there are no neighborly links. An example of such a wheel is given in Figure 4. Note that the network of Figure 1 is not efficient despite being a non-focal wheel as there are some neighborly links in this configuration.

** Figure 4 about here.

Parameter set 4 alters the predictions further and, to a degree, allows the predictions of the efficient and Nash equilibrating principles to be separated. The asymmetric cost structure implies that it is cheaper for agent 1 to connect a certain link than it is for any other agent. This incentive is so strong that the wheel architecture is no longer efficient, and instead a star network centered on agent 1 is the uniquely efficient network, as well as being strict Nash. ${ }^{12}$ Significantly, the wheel network is still a strict Nash equilibrium. The star network is depicted in Figure 5.

** Figure 5 about here.

\subsection{Models of Individual Behavior and Network Dynam- ics}

Since network links reflect individual decisions, such decisions are compelling areas to explore for predicting the existence and behavior of all networks. There are many theories of individual decisions, which become increasingly complex

\footnotetext{
${ }^{12}$ The proofs of these claims are quite simple. To see that the star is a strict Nash equilibrium consider firstly agents 2-6. All of these agents are receiving all pieces of information at the cost of a single link from the cheapest source. Thus, they are playing a strictly optimal strategy. Now consider agent 1 . He is receiving all pieces of information but at the expense of five links (recall he must pay the adjustment fee). However, if he dropped a link then he would lose a piece of information. Thus, he is also strictly optimizing and the star is a strict Nash equilibrium.

To see that this configuration is uniquely efficient suppose that there are links that do not include agent 1 . Say agent 4 is connected from 5 . This link costs $\$ .20$. Consider an alternative network in which this link is omitted and replaced by a link from 5 to 1 and from 1 to 4 . These links cost at most $\$ 0.10$ (as they may already exist). Therefore, this alternative network is cheaper and weakly increases information flow. Consequently, the original network cannot be efficient. It is easy to see that networks involving a subset of links in the star network are also inefficient (just add links of the star that are missing). Therefore, the star centered on agent 1 is uniquely efficient.
} 
in the network environment. We will focus here on two such models, involving varying degrees of strategic choice: (Nash) best response and simple strategic behavior.

Best response, studied in a network context by Bala and Goyal (2000), assumes that agents naively and myopically respond to the network environment. More formally, in a model of simultaneous choice, this decision rule supposes that each agent reacts to the current link selections of other agents by choosing the set of links that maximizes his payoff (given these links). It is myopic in that future payoffs are ignored, and naive in that adjustments by other agents are not anticipated.

Bala and Goyal (2000) develop a model of dynamic network formation involving repeated rounds and in each round agents make simultaneous link formation decisions. They assume that agents best respond although with a degree of inertia (i.e., with some probability they do not change their selection from one round to another). In a remarkable theoretical result, Bala and Goyal (2000) show that, despite the myopic and naive behavior of agents, Nash equilibrium social communication networks (of the one-shot game) evolve very rapidly. This result is perhaps best interpreted as a benchmark with respect to the evolutionary capabilities of networks: that with self-interested and boundedly rational agents convergence to stable networks is possible.

Simple Strategic Behavior is a model based on the possibility that agents act with a greater degree of sophistication than allowed for by the best response decision rule. It may be suspected that agents make choices with more foresight, as well as learn and even teach optimal strategies to themselves and other agents. Unfortunately, given the complexity of network environments, even the simple structure studied here, the application of complex decision rules does not provide much insight or testing power. Therefore, we consider here only one simple decision rule tailored to the network environment. Simple Strategic Behavior (SS) requires agents to connect only one link, and that this link be their part of a focal wheel network. We denote the behavior by (SScc) when the network is the counter-clockwise wheel, and (SScw) when the network is the clockwise wheel.

The logic behind the $\mathbf{S S}$ decision rule is the following. For many parameter values, including sets 1 and 2 from Section 2.2, the wheel network is not only optimal for the agents as a collective, but it is also optimal for every agent individually. Further, the clockwise and counter-clockwise wheels are in many respects focal. Therefore, a reasonable expectation would be that agents are moving towards these configurations even if the corresponding link selections 
are not in their short term interests. These choices would increase the chances of coordination on an optimal network, as well as teach other agents the optimal strategy. ${ }^{13}$ These calculations may not necessarily lead an agent to conform to SS behavior as, for example, he may add an additional link for insurance purposes. However, simple strategic behavior captures the basic intuition of these arguments and intentions, and as we will see later, performs well in describing the choices of agents in network environments.

\section{$3 \quad$ Experimental Procedures}

A total of twelve experiments were performed. Each experiment consisted of six inexperienced subjects recruited from the undergraduate and graduate population of the California Institute of Technology. As summarized in Tables 3 and 4 the experiments consist of five experiments in Series One and seven experiments in Series Two, and followed the design principles described in Section 2.1 .

$$
\text { ** Tables } 3 \text { and } 4 \text { about here. }
$$

Subjects were randomly assigned to locations so friends arriving together tended not to be sitting next to each other. Each subject was assigned an identification number from 1 to 6 . Instructions were read to subjects (see Appendix) and the subjects were given a practice exercise (without payment) and tested before the experiment began.

The experiments consisted of rounds during which subjects could make connections to any other subject at a cost. The profits to a subject for each round were the value of the information received minus the cost of connection. The network began anew at the beginning of each round and links were chosen.

A principle focus of our experimental design is to determine whether decentralized networks could self-organize and converge to stationary configurations. The parameters and procedures of Series One were aimed at exploring this question. The design of Series Two reflects the experiences of Series One. An important operational question in this work is the definition of a stationary configuration. Trading off empirical certainty with experimental constraints, we deemed a network to have "converged" if the same configuration was chosen in three consecutive rounds. ${ }^{14}$ As will be discussed later, in some cases when a

\footnotetext{
${ }^{13}$ This notion is similar in spirit to recent work on "strategic teaching" by Camerer, Ho and Chong (2002).

${ }^{14}$ The experimental constraints included end-effects and potential boredom.
} 
configuration was unchanged for three periods, parameters were changed to see if the "disequilibrated" configuration would evolve to another similar, stationary configuration.

\subsection{Series One}

The experiments of Series One were heavily influenced by the model presented by Bala and Goyal (2000). The startling findings of Bala and Goyal are that decentralized agents can, via a series of simultaneous decision making rounds, organize and stabilize at strict Nash network configurations. In Series One we attempted to test the first half of these findings - that networks can converge to stationary configurations. The experimental design replicated the principle features of the Bala and Goyal model, the primary restriction being that communication among agents was limited solely to their link selections each round (which were announced simultaneously). Parameter set 1, which satisfies the assumptions of Bala and Goyal (2000), was used in all Series One experiments (see Table 1). These values were employed as the confluence of theoretical predictions gave convergence "its best shot" (the wheel is uniquely efficient and strict Nash, as well as focal, network architecture).

The experiments were performed manually and payoffs were calculated using a physical process. In each round every agent recorded their link selection and this was submitted to the experimenter. They then placed in front of themselves, in full view of all agents, physical signs corresponding to their selections. The benefits of connections from the networks were then easily computed with each individual adding the signs exhibited by each node to which the individual was connected. This process quickly iterated to an accurate computation of the information accruing to each node. The network chosen was then drawn on the board at the front of the room. Agents computed their earnings and the round was complete. A random stopping rule was employed to minimize last round effects whereby between 10 and 20 rounds were possible. ${ }^{15}$ There was an increasing chance of stopping as more rounds were played. We refer to this rule as stopping Rule 1. The probabilities of stopping at any point, along with

\footnotetext{
${ }^{15}$ The only exception is experiment 010528 that instead involved a fixed 10 rounds. This trial was included in the final analysis as it provided an additional 60 observations (6 agents, 10 rounds) of individual decisions for tests of behavioral strategies. Critically, the inclusion of this experiment does not favorably bias our results towards network convergence as this experiment did not converge to a stationary configuration.
} 
those for Rule 2 which was used in Series 2 experiments, are detailed in Table 5 .

** Table 5 about here.

\subsection{Series Two}

Several changes were made to the design for Series Two. In this series we attempted to confirm the findings of Series One (convergence to the wheel network) as well as differentiate among the principles that may dictate the convergent state of a network. Parameter sets 2, 3 and 4 were employed. The nonanonymous and asymmetric parameters of sets 3 and 4 allow the predictions of the network principles to be distinguished and, therefore, tested. We expected that coordination problems (which were even present in Series One experiments) would complicate convergence and weaken the test. To facilitate convergence, and thus allow the network principles to be distinguished, we allowed agents to make their link decisions continuously over two minute rounds and for these decisions to be public knowledge and adjusted repeatedly in real time. Previous market experiments as well as committee experiments suggested that this would work.

To operationalize this design the experimental process was moved to computers for Series Two and agents were partitioned into different segments of the laboratory (which further reduced the focalness of the clockwise and counterclockwise wheel networks). The link connection fee was charged, and benefits accrued, only at the end of periods. To minimize cheap talk the agents were charged an adjustment fee of 5 cents each time they added or subtracted a link during each round. All Series 2 experiments commenced with parameter set 2, with the wheel again efficient and a unique strict Nash equilibrium. If convergence was achieved (the same configuration in three consecutive rounds) then the parameters were changed to set 3 and the experiment continued. If convergence was again achieved then parameter set 4 was adopted. Subjects were unaware of the potential change of parameters. ${ }^{16}$

Series Two experiments employed random stopping rule 2, with between 15 and 20 rounds taking place, again with an increasing probability of stopping as more rounds occurred. The details of both experimental series are detailed in

\footnotetext{
${ }^{16}$ Thus, the application of parameter sets 3 and 4 were determined endogenously by play in early rounds of the experiments. The resultant self-selection of treatments is intentional as the question we wish to address is conditional: given a network can converge, which principle determines the convergent state?
} 
Table 6 .

** Table 6 about here.

\section{Results}

The results are divided into three sections. We begin with findings on macro features of network structures, and continue with an investigation of the strategies employed by individual agents. We conclude with synthesis results on how individual decisions impact the evolution of dynamic networks.

\subsection{Macro: Network Configurations}

Table 7 contains a summary of data from all experiments. Eight of the twelve networks converged to Nash equilibrium configurations (two of five from Series One and six of seven from Series Two). The convergent state was achieved as early as round 4 and as late as round 17. All convergent states were Nash equilibria of the one-shot game, although not always strict Nash. The remaining four experiments did not converge to any stationary configuration, Nash or otherwise but three of these experiments temporarily achieved Nash configurations (either weak or strict) that did not prove stationary. At no point in any experiment was the empty network chosen.

** Table 7 about here.

The first result is that networks can occur and evolve. Agent decisions reflect the unique characteristics of networks as opposed to arbitrary choices.

Result 1 Networks happen. Not only are links formed but an appreciation of the externalities inherent in networks is incorporated into agent decisions.

Support: In each experiment a network instantaneously formed. At no point was the empty network nor the complete, point-to-point network chosen (i.e., everyone connects to everyone else) reflecting understanding of the value and externalities in link formation.

This basic evidence suggests that given appropriate conditions a social or economic network will emerge. The simple observation provides initial confirmation that networks can arise by economic forces. In the remainder of the paper we attempt to understand the nature of these economic forces. 
In a sense Result 2 is central by establishing two important facts. First, the process of network formation tends to stop - a type of equilibration. Secondly, the final configuration tends to be at a Nash equilibrium. Thus, there is a convergence process and the forces at work in the process are captured by game theory in general and the Nash equilibrium in particular. Network formation is not simply a random process.

Result 2 (a) Networks tend to converge to stationary configurations, and (b) Nash equilibrium is a necessary condition for stationarity, and (c) a greater tendency toward convergence is exhibited by institutions that allow continuous adjustment.

Support: (a) See Table 7. Eight of the twelve networks converged to Nash equilibrium configurations (two of five from Series One and six of seven from Series Two). After convergence, the parameters were changed in three of the Series Two experiments and convergence to different networks followed in all three. The convergent state was first achieved in rounds 9 and 11 of the Series One experiments, and in rounds 17, 16, 5, 7, 4, and 15 of the Series Two experiments.

With six agents there are $\left(2^{5}\right)^{6}=1,073,741,824$ possible networks. The probability of convergence with random selection in an $n$ round experiment (the same network in three consecutive periods) is then strictly less than $\frac{n-2}{\left(2^{30}\right)^{2}}{ }^{17}$ Therefore, the hypothesis that network dynamics are random can be rejected with an extremely high level of confidence. ${ }^{18}$

(b) All eight convergent networks (and the three re-convergent networks after parameter changes) are Nash equilibria of the one shot game. In no experiments did a network exhibit equilibration at non-Nash equilibrium configurations.

(c) Continuous decision making was employed in Series Two experiments and discrete decision making was employed in Series One. Roughly speak-

\footnotetext{
${ }^{17}$ This simple expression is the probability that any three consecutive networks are identical in $n$ periods. It is used here for analytical simplicity. The exact probability that the experiment ceases because of convergence is strictly less than this.

${ }^{18}$ It should be noted that the claim that network dynamics are not random is quite robust. Even if we restrict agents to choose only one link at a time (what they would need to choose in the efficient Nash network) then randomness can still be rejected at a high level of significance. In this case there are $5^{6}=15625$ possible networks. Thus the probability of convergence with random selection in an $n$ period trial is strictly less than $\frac{n-2}{15625^{2}}$.
} 
ing, continuous decision making seemed to aid convergence (convergence in six out of seven experiments versus 2 out of 5 ).

While convergence is not guaranteed, the predictability of convergence, in addition to the convergence itself, should be interpreted as strong evidence that something systematic is driving network dynamics. Clearly the Nash equilibrium is a useful concept for capturing what is observed and that fact suggests questions about other features of the model and other principles that might be used in conjunction or as substitute principles for modeling and understanding the process. Three concepts surface immediately. Two of these, efficiency and focalness, were detailed in Section 2. The third possibility is the concept of strict Nash equilibrium, a refinement of Nash equilibrium. In the many applications of game theory it is well known that the concept of Nash equilibrium is a somewhat weak condition. These same concerns apply to the study of networks as well. Bala and Goyal (2000, p.1194) calculate that there exist in excess of 20,000 Nash networks for the environment studied in this paper. They show that the refinement of strict Nash equilibrium reduces the equilibrium set to a unique architecture (the wheel) which has 120 possible configurations. Our next result provides evidence that none of the three alternative possibilities accurately predict stationary network configurations.

Result 3 Each of focalness, efficiency, and strict Nash equilibrium can be rejected as being a necessary condition for a configuration to become stationary.

Support: Experiment 010607b converged to a non-focal and inefficient configuration in rounds 16-18. Experiment 010528 converged to a non-focal wheel in rounds 17-19. Further, after the parameter changes in experiments 010613a, 010613b, and 010614a, the networks diverged from the focal wheel (that was no longer efficient) and reconverged to non-focal wheels. In experiment 010607b the network converged to a weak Nash equilibrium configuration. This convergent network is depicted in Figure 6 . In this network agent 5 is indifferent between connecting a single link from agents 1,2, 3 or 6 , and agent 3 is indifferent between connecting a single link from agents 4,5 , or 6 .

** Figure 6 about here. 
Combined with Result 2, this result indicates that Nash equilibrium is the guiding principle of network dynamics and convergence, and that focalness and efficiency are not. ${ }^{19}$ This result confirms, if nothing else, that networks are a real economic phenomenon, and should be looked at from an economic perspective. Networks exhibit the classic economic tension between individual incentives and inefficient outcomes.

An important caveat to Result 2, and further complicating the question of an appropriate model, especially one with roots in equilibrium selection concepts, is that Nash configurations did not always prove stationary in the different experiments. We observe that network formations can "pass through" Nash equilibria.

Result 4 The principle of Nash equilibrium, or even strict Nash equilibrium, does not prove a sufficient condition for a configuration to be stationary.

Support: Weak Nash configurations that did not prove stable were played in experiments 981106 (two weak Nash), 010528, 010607a, 010607b, 010613a (three weak Nash), and 010614b. Further, strict Nash configurations (the wheel) were played in experiments 990115, 010607a (in rounds 4, 11-12, and 15), and 010614b (in rounds 9-10, and 12-13). In experiment 010614b the same strict Nash configuration played in rounds 9-10 and 12-13 ultimately proved stationary in rounds 15-17.

In view of Result 2, these deviations, particularly from the strict Nash configurations, are surprising and naturally lead to speculation and conjectures about how the model might be modified to account for the phenomena. The most obvious candidates are that these deviations resulted from mistakes, boredom, or confusion. However, this would not seem to be the complete story for the following reasons. Firstly, all participants successfully completed the example calculations in the instructions. Secondly, at least in Series 2 the participants had the opportunity to rectify any mistakes. ${ }^{20}$ And, thirdly, no

\footnotetext{
${ }^{19}$ Although the evidence supporting Result 3 is brief, it is sufficient to provide counterexamples and support the claim. Further evidence against efficiency is provided in Result 5.

${ }^{20}$ Assuming they were not making their choices at the last second. This was only the case for one agent in experiment $010614 \mathrm{~b}$ and this agent, in fact, was not the one to deviate from the Nash equilibrium.
} 
participants indicated any of these three factors in their comments at the end of the experiments. ${ }^{21}$

Deeper speculations lead to the idea of common knowledge upon which the notion of equilibrium is built. With respect to equilibrium this concept says that every agent knows that every agent is maximizing, and that every agent knows every agent knows every agent knows, and so on. Consequently, it is possible that a group of agents is not in a stationary network even though the focal, efficient and strict Nash wheel configuration is being played. Supporting evidence, although weak, can be found in experiment 010614b where agent 3 delayed his link choice in each round of play until only a few seconds remained, even when a focal wheel was reached in rounds 9-10. This behavior suggests that agent 3 was not completely aware of the strategic situation, and may have been a factor in the agent 5's deviation from Nash in round 11. If this was in fact how agent 3 was playing then a notable finding is that efficiency and coordination were still achieved with individually optimizing behavior. This possibility is consistent with the intuition behind Bala and Goyal's result.

We conclude this section with an important finding on the dynamic path of networks (we return to dynamics in Section 4.3). Result 5 asks if "efficiency seeking" alone, which is closely related to the Nash equilibria, could be driving the results to Nash. By looking at the non-convergent examples and asking if they are efficiency improving even if they do not converge to some stationary configuration, the question is answered negatively.

Result 5 Non-convergent networks do not exhibit increasing efficiency.

Support: See Figures 7 a, b, and c, and Figure 8b. These graphs represent measures of network efficiency throughout experiments 981106, 990115, 990128, and 01067a, respectively (the non-convergent networks). The measure of efficiency in a network is the amount of information earned per link paid for in the network as a whole. So if the network is at an efficient wheel configuration the measure of efficiency is 6: each agent receives every available piece of information at the cost of only one link. The slope parameters of these graphs were estimated using ordinary least squares and the t-statistics of these estimates are, respectively, 0.47, 1.74,

\footnotetext{
${ }^{21}$ These comments were only elicited after the experiments of Series Two. As a result of the deviations from Nash configurations in the experiments of Series One we began asking participants to describe, at the completion of the experiment, the strategy they employed as well as how they thought their fellow participants were behaving.
} 
1.33, and 1.08. So for all four experiments we fail to reject, even at the $10 \%$ level, the null hypothesis that efficiency is not increasing.

** Figures 7 and 8 about here.

Result 5 makes an important point. It tells us that the dynamics of network evolution and change are not guided by a principle of efficiency seeking (and thus inefficiencies can be institutionalized). This suggests that the principles that are work are not the same as those in markets, where trade tends to guide the process to increasing levels of efficiency. Instead the principles seem to have elements of public economics, where unilateral actions do not necessarily increase efficiency unless they are executed within some carefully crafted mechanism.

Note that Results 3 and 5 do not imply that efficiency and focalness should be completely disregarded in analyzing networks. While they are not the determining principle when considered alone, the appropriate model might include elements of each - some combination of these principles. Indeed, the fact that six out of eight convergent networks were to the focal wheel suggests the possible importance of theoretical mergers.

\subsection{Micro: Individual Decision Making}

In our attempt to understand the evolution of networks we turn now to individual behavior. The complexity of networks and the relatively few observations we obtain for each agent make the job difficult but we are able to construct significant tests of decision rules from the theoretical literature. We also test a conjecture, described in Section 2.3, that in a dynamic environment agents will use link choice to signal and teach, other agents. In the following section we attempt to piece together behavioral findings with the dynamics of Section 4.1 to further understand the evolution of networks.

In this section we restrict attention to the decisions of individuals in the experiments of Series One. This is done for two reasons. Firstly, this series most closely resembles the theoretical model of Bala and Goyal (2000) and, therefore, provides the more appropriate test of their theory of individual behavior. Secondly, it provides, in a sense, cleaner data. In Series Two agents made decisions continuously and it is difficult to infer the information available to, or the intentions of, each agent at the time the decision was made. 
To test and compare decision rules we employ statistical techniques introduced by El-Gamal and Grether (1995). Decision rules are generally modeled as rigid and precise behavior and are easily rejected by a single deviant. To allow for noise in data, El-Gamal and Grether suppose that the use of any particular decision rule is prone to error of a given probability (whether by the agents themselves or external factors). The likelihood that the decision rule (now with a fixed error rate) produced the observed sample is then estimated. Essentially the test involves an examination of how frequently the decision rule was used (e.g., the more it was used then the more likely it could, for a given error rate, produce a given sample). If the likelihood thus calculated is too small then we reject the hypothesis that the decision rule with error was used by the agent. By examining the likelihood that different decision rules produced an observed sample, these techniques also allow us to determine which rule is more likely to have been employed by a particular agent.

The first test is of the best response decision rule employed by Bala and Goyal (2000) (see Section 2.3). The best response rule is not well supported by the data from the decisions of individual agents. We find that no agent follows best response precisely, and that only weak evidence exists that agents are even best responding with error. The lack of accuracy of the best response model leads naturally to models of individual decisions that are somewhat more sophisticated but also a bit more ad hoc. We compare best response to one such strategy, simple strategic behavior, and find that a majority of agents are more likely to be using the counter-clockwise simple strategic decision rule. Further, we find the evidence supporting best response is further weakened once attention is restricted to agents more likely to be employing the rule. The full sample support for best response (albeit weak) arises only because the best response and simple strategic decision rules coincide in their requirements for some network configurations. That is, what appears as best response behavior is indistinguishable from simple strategic behavior.

Result 6 (i) Agents do not act in accordance with the best response decision rule,

(ii) More agents exhibit counter-clockwise simple strategic behavior than best response. Moreover, among agents more likely to be using best response, very weak evidence supports that they are actually using best response.

Support: (i) As a deterministic decision rule best response is rejected immediately as no agent followed its requirements every round, even allowing 
for any degree of inertia. The left half of Table 8 presents the findings for all thirty agents when allowing for error. As inertia was originally included to model non-optimal behavior it has been included as an error in the analysis.

** Table 8 about here

The hypothesis that best response is being employed can be rejected. Weak support is only available for a $25 \%$ error level, although this result is more reflective of the lack of power of the tests than confirmation of the decision rule.

Aggregating the data leads to an even more overwhelming rejection. We find that even with a $50 \%$ chance of error the hypothesis that agents employ the best response decision rule with error can be rejected at the $1 \%$ level of significance. Such an aggregated test is appropriate if, as mentioned previously, agents are assumed to be homogenous. Therefore, either no agent uses the rule or the assumption of homogeneity across agents is inappropriate.

(ii) 12 agents are more likely to be using best response rather than simple strategic behavior. One agent is equally likely to be using both rules. The right half of Table 8 restricts the analysis of the left side of the table to only the $12 \frac{1}{2}$ agents more likely to be using best response.

This evidence on individual behavior contrasts with the findings of the previous section that networks are indeed capable of converging to the efficient and Nash equilibrium wheel network. The systemic behavior is predicted by the model of Bala and Goyal, but the evidence at the individual level conflicts with the micro behavior postulated by their model. This combination leads to a paradox frequently observed in economic experiments that the models work well when applied at the systemic level but the exact behavior of the agents is at odds with the behavioral principles at the foundation of the model.

We turn now to a detailed analysis of simple strategic behavior. The following result indicates that the strategy and foresight underlying simple strategic behavior is evident within networks.

Result 7 (i) Many agents exhibit significant simple strategic behavior, some agents use simple strategic behavior in every round,

(ii) More agents exhibit simple strategic behavior than best response. Agents using simple strategic behavior are more likely to follow the decision rule than agents best responding. 
Support: (i) Despite the fact that a wheel network appeared in only nine out of the 67 rounds played in the five experiments of Series One, five out of the thirty agents exhibited simple strategic behavior in every single round. Two of these agents persisted with this strategy despite participating in experiments in which a wheel network never occurred. Three of the five agents in fact selected the same link, the counter-clockwise wheel (SScc), every period of their experiment. The other two changed between the clockwise and the counter-clockwise wheels at some point in the experiment in what appears to be attempts to coordinate with other agents on which of the focal wheels will actually be chosen. The remaining agents do not act consistently and uniformly in accordance with simple strategic behavior. So, it is a question of frequency and propensity. Table 9 and the left half of Table 10 present the findings on whether agents exhibit simple strategic and counter-clockwise simple strategic behavior allowing for error. As can be seen, many agents appear to act in accordance with simple strategic behavior.

** Tables 9 and 10 about here.

(ii) 17 agents are more likely to be using counter-clockwise simple strategic behavior rather than best response, one agent is equally likely to be using both rules. The right half of Table 10 restricts the analysis of the left side to only the $17 \frac{1}{2}$ agents more likely to be using SScc.

These results indicate that most agents engage in strategic signaling and coordination efforts through their link selections. Relatively few attempt to optimize current payoffs by using the best response decision rule. Further, the results imply that agents behave in some sense strategically and with foresight in network environments. Many agents employ a strategy that seems to be an attempt to teach, signal, and coordinate all agents within a network and in doing so facilitate movement toward Pareto optimality. Not all agents employ the same strategy. It would appear that agents are making decisions on more analysis than simply their immediate payoff or their own actions. ${ }^{22}$

\footnotetext{
${ }^{22}$ These conclusions should not be interpreted as conflicting with the results of Bala and Goyal (2000). As mentioned previously, the results of Bala and Goyal are best interpreted as the benchmark capabilities of network evolution.
} 


\subsection{Interdependence: Micro and Macro}

The findings of the previous section leave two prominent questions. Firstly, how instrumental are the strategies of individuals, particularly those employing simple strategic behavior, in achieving convergence? And, secondly, how do agents behave who neither best respond nor use simple strategic behavior? We produce an answer to the first question, and present some aggregate evidence to provide insight into the second, which is explored with the final result of this section.

The behavior of individual agents appears to be crucial to whether or not convergence to the wheel network is achieved. Specifically, individual agents appear to be capable of influencing the evolution of networks by signaling to their fellow agents an optimal strategy. By following SS behavior agents can teach other agents the structure of the game and the nature of payoffs. This induces these agents, who begin with some other strategy, to switch to SS behavior and as a consequence the probability of convergence increases.

This individual capability is best seen by considering how convergent and non-convergent networks differ. Surprisingly, they differ by only a small, but significant, amount. For all experimental networks, convergent or not, the majority of agents exhibited some simple strategic behavior throughout and several agents did not. In the convergent networks the remaining agents learned the optimal configuration and began coordinating with their fellow agents on counter-clockwise simple strategic behavior. In the non-convergent networks this learning did not occur. These findings are captured by the following two results.

Result 8 Network convergence is critically dependent on the behavior of all agents. Moreover, agents can learn to choose optimal strategies and enable networks to converge to efficient, Nash outcomes.

Support: The results are presented in Table 11. All six agents in the networks that converged exhibit counter-clockwise simple strategic (SScc) behavior. By contrast, for the non-convergent networks such consistency of behavior was not observed, although there were at least four agents for whom randomness is rejected in favor of SScc behavior.

** Table 11 about here.

Upon closer inspection, however, it can be seen that all six agents in convergent networks do not exhibit SScc behavior consistently throughout 
the experiment. To expose this shift in networks that converged, Table 12 performs the same analysis as above but omits the periods after convergence has occurred. It can now be seen that, surprisingly, convergent networks look very similar to the networks that didn't converge.

$$
\text { ** Table } 12 \text { about here. }
$$

Thus, all of the experiments appeared similar up until a critical point in two of the networks at which the remaining agents learned to play the SScc strategy and convergence to efficiency was achieved. In the non-convergent networks this learning simply did not occur, and consequently inefficiency was the result.

The next result shows that the devotion of agents to a particular strategy, in this case SScc, is also critical to network dynamics. Agents in the ultimately stationary networks are significantly more committed to the simple strategic strategy, and this dedication appears to be pivotal in achieving convergence.

Result 9 Network convergence depends on the commitment to simple strategic behavior of individual agents. Thus, all agents can impact the probability of convergence.

Support: This result is exposed by the relationship between whether a network converged and the rate at which counter-clockwise simple strategic behavior is played (before convergence was achieved in the convergent networks). ${ }^{23}$ Table 13 details these variables.

** Table 13 about here.

Unfortunately, because of the small number of observations, the power of this test is limited. Nevertheless, a significant relationship emerges. Estimating the equation $y=\alpha+\beta\left(\frac{\gamma}{n}\right)+\varepsilon$ by OLS (where the variables are defined in the table and $y$ is coded 1 for convergence and 0 otherwise) we find that the estimate of $\beta$ is positive and significantly different from zero ( $\beta$ estimate of 0.61 , t-statistic of 2.38 , and $p$-value of $0.14 ; \alpha$ estimate of -0.97 , t-statistic of -1.51 , and p-value of 0.26$).{ }^{24,25}$

\footnotetext{
${ }^{23}$ If instead the number of observations by the leading four agents is used here, as may be interpreted from Result 10, the conclusions are not affected (the number of observations in this instance are $22,36,20,23$, and 26 , respectively).

${ }^{24}$ For this regression we omitted the rather special case of experiment 990115 that achieved the focal wheel but immediately diverged. Including it in the estimation as a non-converged network (or even a converged network) does not change the results substantially.

${ }^{25}$ As we are interested in establishing the existence of a significant relationship between
} 
These results begin to expose the integral role of individual decisions in network formation. Agents can educate their fellow agents to follow strategies that lead to efficient outcomes but this requires the educator agents to play their component of the focal wheel network, and to play it consistently. Unfortunately the precise nature of the resulting learning cannot be clearly ascertained from these data. The final two agents may learn to play the simple strategic strategy because the repeated play of SScc by the other agents has taught them the common benefits of such play. Alternatively, they may eventually play SScc because it is a best response to the choice of SScc by their fellow agents.

The next result indicates that there is still more to the story of individual behavior and how agents are making decisions if not using SScc. Unfortunately, the possibilities are far too complex for meaningful tests of individual behavior to be conducted on the relatively few observations reported here. As a result, the analysis turns to the aggregate data. In an admittedly crude test, and employing data from both experimental series to increase test power, we are able to infer aggregate responses to network situations which exhibit best responselike behavior.

The logic behind the aggregate analysis, which is reported as Result 10 below, is the following. If in a network the total number of information pieces collected is less than 36 (six pieces per agent) then the network is inefficient as it must be possible to add additional links that would increase individual and group profit. Therefore, the best response of at least one individual, assuming all other links remain unchanged, is to add more links. Similarly, the network is inefficient if the number of links connected is greater then six, and it must be the best response of at least one agent to reduce the number of links connected.

Aggregate level quasi-best responsiveness We say that there is "aggregate level quasi-best responsiveness" if an inefficiency caused by a shortage of information is followed at the aggregate level by the connection of additional links. Likewise, if all information is collected and there is an excess of links then this is followed by a reduction in the number of links.

While aggregate data analysis cannot be used to make inferences about specific individuals, it can be used as a guide to the nature and process of network evolution and as a source of suggestions about models that might be useful at the

these two variables, we will not make a further distributional assumption to produce estimates of the probability of convergence (though, of course, if the uniform distribution is assumed the probability estimates are equal to the constrained OLS estimates). 
individual level. There are many possible explanations for individual behavior that could produce the aggregate effects documented here. Arguably, however, the main lesson suggested is that agents not employing the SScc strategy are still exhibiting some kind of rationality.

Result 10 Network dynamics exhibit strong evidence of "aggregate level quasibest" responsiveness.

Support: Evidence of this group dynamic can best be seen graphically. Figure 9 depicts the relationship between the total number of links selected and the average number of pieces of information received by each agent through the 18 rounds of experiment $010607 \mathrm{~b}$.

** Figure 9 about here.

The aggregate level quasi-best responsiveness is evident in the correlation of these two measures. In all but one period (the final period before convergence) an inefficient accumulation of information by the agents (less than 6) is followed by an increase in the number of connected links in the following rounds. Similarly, in all rounds (other than after convergence was achieved) an efficient accumulation of information by the agents (average of 6 ) is followed by a decrease in the number of connected links in the following rounds.

To test this idea more formally we regress the average information value on the change in total links from period to period. This is written,

$$
\Delta_{t}=\alpha+\beta I_{t}
$$

Where $I_{t}$ is the average information level in round $t, \Delta_{t}=L_{t+1}-L_{t}$, and $L_{t}$ is the total links chosen in round $t$. The estimates for this equation for all experiments combined are given in Table $14 .^{26}$

** Table 14 about here.

These findings indicate that the causal relationship between information accumulation and changes in the number of links selected is negative and statistically significant (at the $1 \%$ level). The data produce strong support for aggregate level quasi-best responsiveness.

\footnotetext{
${ }^{26}$ For consistency, rounds in Series Two after convergence (when the parameters were changed) are excluded.
} 


\section{Conclusion}

This research has attempted to present some key characteristics and principles of network evolution. The principles studied are theoretically general, with potential applications beyond the particular environments studied here. From a theoretical point of view there are many reasons why decentralized network development and evolution might fail. The flows within networks create externalities and, not surprisingly, free rider issues that relate to the public goods problem. Similarly the development of these links involve coordination problems and, as they are typically costly, implicit bargaining. Indeed, the asymmetry of payoffs within networks, even efficient and Nash equilibrium configurations, is reminiscent of ultimatum games (all agents force another into a low payoff network).

In spite of the inherent potential for problems, not only do networks develop (Result 1) but they tend to converge to a configuration that has the properties of a Nash equilibrium (Result 2). While Nash appears to be a necessary condition for stationary configurations, efficiency and Strict Nash do not (Result 3). Furthermore, none of the candidate models are sufficient conditions for convergence (Result 4) and the dynamic paths are not efficiency enhancing (Result 5).

The analysis shifts focus from an equilibrium and stationarity perspective to a focus on the decision rules and behavior exhibited by the individuals. Individuals tend to use simple strategic responses as opposed to Nash responses (Results 6 and 7). This creates a paradox not atypical of economic models. The equilibrium model receives support but the model of individual decisions on which the equilibrium model is created does not. This paradox caused us to look somewhat deeper into the data to see what might be suggested. Two prominent features reveal themselves. First, network convergence is associated with strategy commitments by some agents (Results 8 and 9) and this commitment could play a role of "teaching" other agents about "appropriate" behavior. Secondly, the system exhibits a response to events at an aggregated level (Result 10). In particular, if the system "needs" additional links in a period the aggregate response is an increase in the number of links the next period. Similarly if the system has too many links then in the aggregate the number of links is reduced. Of course these responses are themselves uncoordinated so may or may not improve efficiency.

Basic lessons emerge. The first is that the recent models of network emergence have predictive power but the source of that power is not entirely clear 
and may be fragile. That is, while the environments studied here would seem to be very supportive of convergence, many parameter changes including asymmetric costs and information, decay, lack of public knowledge of links or moves, multiple directional flows, etc. can produce networks of much greater complexity than we have studied. Furthermore fragility might be related to the types of institutional arrangements within which network formation takes place. The results reported here suggest that institutions that facilitate an understanding of the intentions of players, a means for formation of and communication of rationality and a common knowledge of it, could be important.

\section{Appendix}

\section{NETWORK EXPERIMENT INSTRUCTIONS ${ }^{27}$}

This is an experiment of network formation. If you follow these instructions and make appropriate decisions, you can earn an appreciable amount of money. At the end of the experiment your earnings will be paid to you privately, and in cash. In this experiment each person holds some private information. This information is valuable to you, and to every other person who can access it. It has a value of 25 cents for every person, including yourself, who holds it. You can access someone else's information directly by forming a link from them. Each link costs 15 cents. You may form as many links as you like. A piece of information can be passed along multiple times.

Say in a three person network that you connect a link from Person 1. Then if Person 1 is also linked to Person 2 you receive the information from both Person 1 and Person 2, but you only have to pay for the link from Person 1. This is shown in the following diagram. Note that the tip of the arrow points to the person who is paying for the link and so receiving the information.

\footnotetext{
${ }^{27}$ These are the instructions used in Series Two of the experiments. Other than minor changes to allow for the differences between the series, the instructions used in Series One are identical.
} 


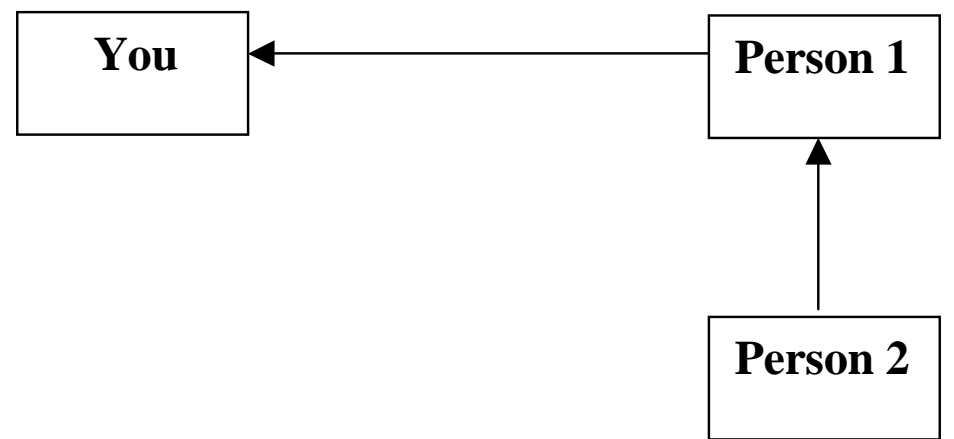

Information you now hold: You, Person 1, and Person 2

Value $=3 \times 25$ cents

Costly links you have paid for: From Person 1

Cost $=15 \mathrm{c}$

Net profit for you for this round $=75 \mathrm{c}-15 \mathrm{c}=60$ cents

The information links are only one way. So in the above example, if you have paid for the link from Person 1 then you receive Person 1's information, but Person 1 does not receive your information. Person 1 would have to pay for a link from you if he wanted your information. Note that it is permitted for any two people to pay for links from each other simultaneously.

Let's try a simple example. In the following diagram what would be the profits for persons 1,2 and 3? Write your answers in the space provided. Represent links paid for by circling the person number from whom each link is connected. Circle ' $\mathrm{N}$ ' if a person has not paid for any links. Remember, you also receive value from holding your own information.

\begin{tabular}{|c|c|c|c|c|}
\hline \multirow[t]{3}{*}{ Person 1} & & \multicolumn{2}{|c|}{ Person 2} & \\
\hline & Person 3 & & & \\
\hline & Links Chosen & Cost & Info & Profit \\
\hline Person 1 & N 123 & $15 \mathrm{c}$ & & $60 \mathrm{c}$ \\
\hline Person 2 & N 123 & & & \\
\hline Person 3 & N 123 & & & \\
\hline
\end{tabular}


The experiment will involve multiple rounds. Each round will close after two minutes. In each round you will select from which people you wish to pay for a link. You will mark your selection(s) in the box marked 'Link Submission Form' and submit by clicking on 'submit links.' You may change your selections as often as you like during each round. However, you will be charged an adjustment fee of $5 \mathrm{c}$ every time you add or subtract a link and click on 'submit links.' (5c for each addition or subtraction) You can record these charges on your Record Sheet in the column marked 'Cost of Changes.'

The box marked 'Connections' represents the links currently selected by each person (read horizontally), with your expected payoff below. This record will be updated continuously as people connect and disconnect links during each round.

The 'Link/Total Value' box describes the value you will receive by connecting a single link from each other person. At the end of each round you will pay the $15 \mathrm{c}$ connection fee for each currently selected link. These connections will then be used to calculate the information you accumulate and the earnings you receive. Note that you will be paid for your performance in each round of play.

This process is then repeated in each subsequent round. In each round connections start anew, so you will pay for any links you hold in that round, regardless of whether you have held that link previously. In each round you may connect any link, or combination of links, that you desire.

The exact number of rounds to be conducted will be determined randomly. We will conduct at least fifteen rounds. At the end of the fifteenth round and after every subsequent round a pair of dice will be rolled. If the sum of the roll exceeds a certain number, specified in the table below, then the experiment will stop. Otherwise we will continue with another round and repeat the process. You will notice that the probability of stopping after a given round increases as we play more rounds.

\begin{tabular}{|c|c|c|}
\hline Round & End if Sum $\geq$ & Prob End \\
\hline 15 & 12 & $1 / 36$ \\
\hline 16 & 10 & $6 / 36$ \\
\hline 17 & 8 & $15 / 36$ \\
\hline 18 & 6 & $26 / 36$ \\
\hline 19 & 4 & $33 / 36$ \\
\hline 20 & 2 & 1 \\
\hline
\end{tabular}

After the experiment is completed you will be paid your profits. Are there 
any questions before we begin? Please do not talk or communicate with anyone else during the experiment. We will insist that everyone remain silent until the end of the last period. If we observe you communicating with anyone, other than the experimenter, we will ask you to leave without completing the experiment. We are now ready to begin round one. Please choose your desired connections for round one on your screens.

\section{References}

[1] Bala, V. and S. Goyal (2000). "A Noncooperative Model of Network Formation." Econometrica 68 (September): 1181-1230.

[2] Bala, V. and S. Goyal (2000a). "A Strategic Analysis of Network Reliability." Review of Economic Design 5: 205-228.

[3] Calvo-Armengol, A. and M. Jackson (2003). "The Effects of Social Networks on Employment and Inequality." American Economic Review, forthcoming.

[4] Camerer, C.F, T-H. Ho, and J-K. Chong (2002). "Sophisticated Experience-Weighted Attraction Learning and Strategic Teaching in Repeated Games." Journal of Economic Theory 104 (May): 137-188.

[5] Dutta, B. and S. Mutuswami (1997). "Stable Networks." Journal of Economic Theory 76: 322-344.

[6] El-Gamal, M. and D. Grether (1995). "Are People Bayesian? Uncovering Behavioral Strategies." Journal of the American Statistical Association 90 (December): 1137-45.

[7] Hendricks, K., M. Piccione and G. Tan (1999). "Equilibria in Networks." Econometrica 67 (November): 1407-1435.

[8] Jackson, M. (2003). "A Survey of Models of Network Formation: Stability and Efficiency." Group Formation in Economics: Networks, Clubs, and Coalitions, ed. G. Demange and M. Wooders, Cambridge University Press (Cambridge, UK), forthcoming.

[9] Jackson, M. and A. Watts (2002). "The Evolution of Social and Economic Networks." Journal of Economic Theory 106 (October): 265-295. 
[10] Jackson, M. and A. Wolinsky (1996). "A Strategic Model of Economic and Social Networks." Journal of Economic Theory 71: 44-74.

[11] Keren, M. and D. Levhari (1983). "The Internal Organization of the Firm and the Shape of Average Costs." Bell Journal of Economics 14: 474-486.

[12] Kranton, R. and D. Minehart (2001). "A Theory of Buyer-Seller Networks." The American Economic Review 91 (June): 485-508.

[13] Ledyard, J. (1995). "Public Goods: A Survey of Experimental Research." The Handbook of Experimental Economics, J. Kagel and A. Roth (ed.). Princeton University Press (Princeton, New Jersey).

[14] Montgomery, J. (1991). "Social Networks and Labor Market Outcomes: Toward an Economic Analysis." American Economic Review 81: 14081418.

[15] Neumark, D. and A. Postlewaite (1998). "Relative Income Concerns and the Rise in Married Women's Employment." Journal of Public Economics 70: $157-183$.

[16] Schelling, T.C. (1960). The Strategy of Conflict. Harvard University Press (Cambridge, Massachusetts).

[17] Wellman, B. and S. Berkowitz (1988). Social Structure: A Network Approach. Cambridge University Press (Cambridge, Massachusetts). 


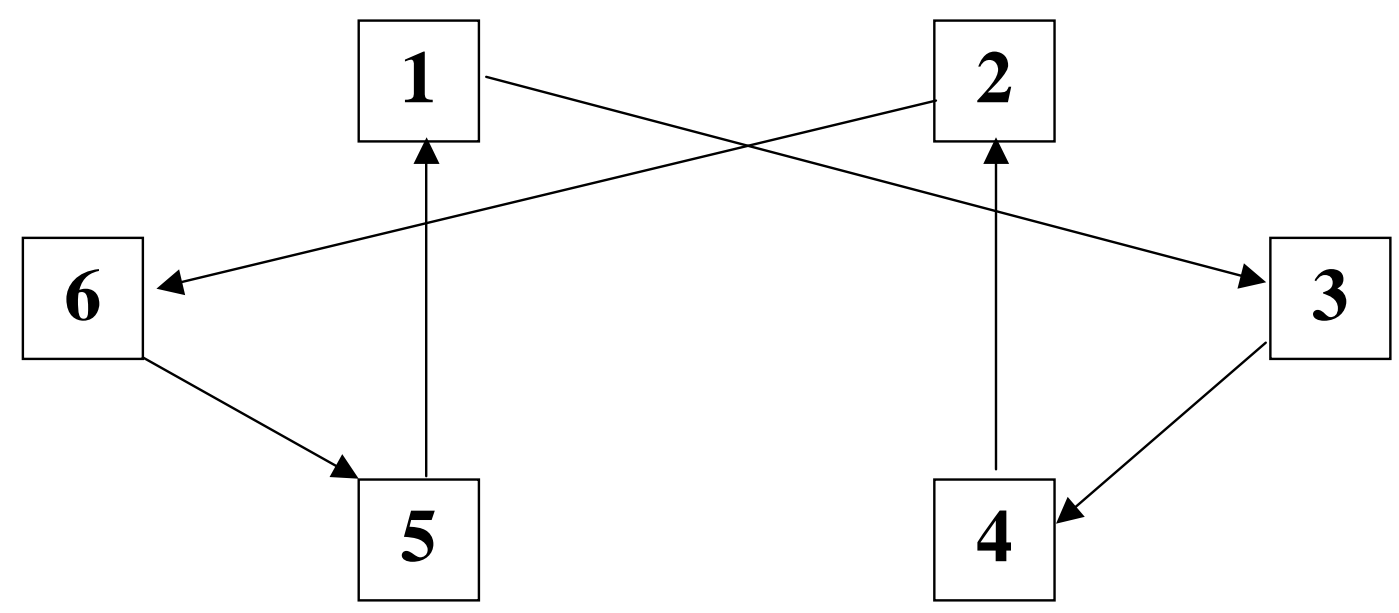

Figure 1: A Six Agent Network

\begin{tabular}{|lll|}
\hline $\begin{array}{l}\text { Parameter Set } \\
\text { Number }\end{array}$ & Link Connection Cost & $\begin{array}{l}\text { Info Value } \\
\text { per unit }\end{array}$ \\
1 & $\$ 0.15$ & $\$ 0.20$ \\
2 & $\$ 0.15$ & $\$ 0.25$ \\
3 & $\$ 0.30$ from neighbors, $\$ 0.15$ from others & $\$ 0.25$ \\
4 & free connection in/out $1, \$ 0.15$ from others & $\$ 0.25$ \\
\hline
\end{tabular}

Table 1: Parameter Sets

\begin{tabular}{|lllll|}
\hline Parameters & Strict Nash & Weak Nash & Focal & Efficient \\
Set 1 & wheel & many (e.g., star) & (counter-)clockwise wheel & wheel \\
Set 2 & wheel & many (e.g., star) & (counter-)clockwise wheel & wheel \\
Set 3 & wheel & many (e.g., star) & (counter-)clockwise wheel & non-focal wheel \\
Set 4 & wheel/star & many & (counter-)clockwise wheel & star centred on 1 \\
\hline
\end{tabular}

Table 2: Model Predictions 


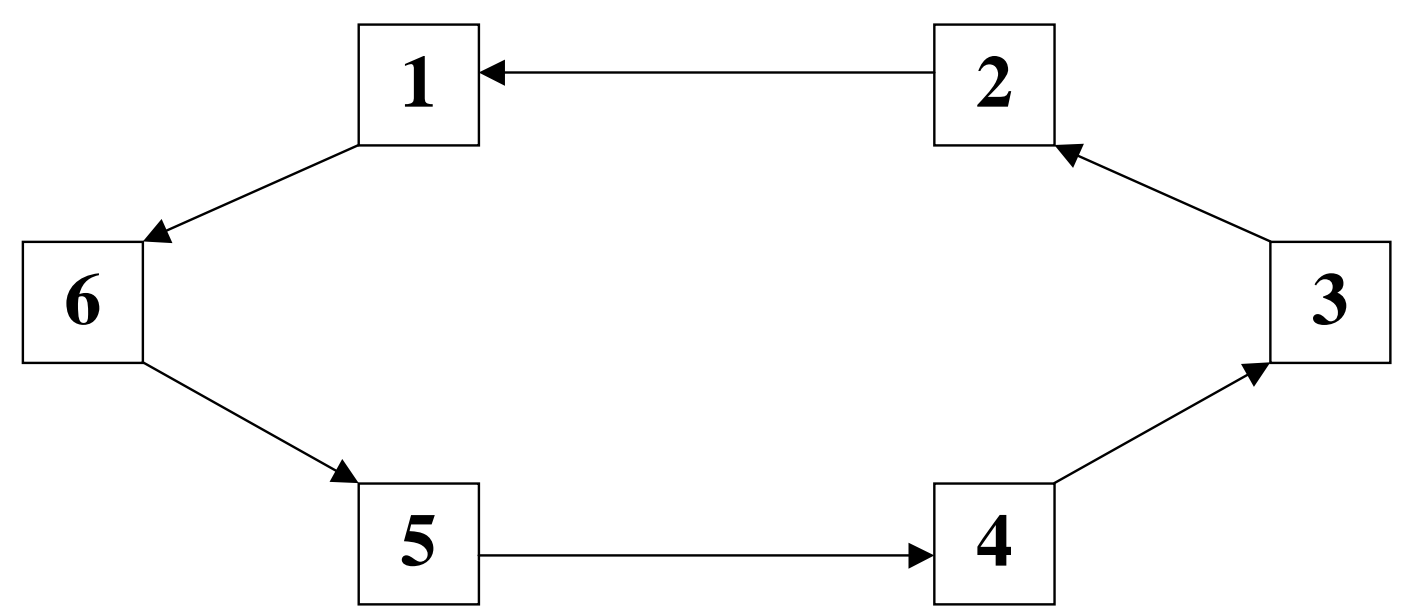

Figure 2: The Counter-Clockwise Wheel

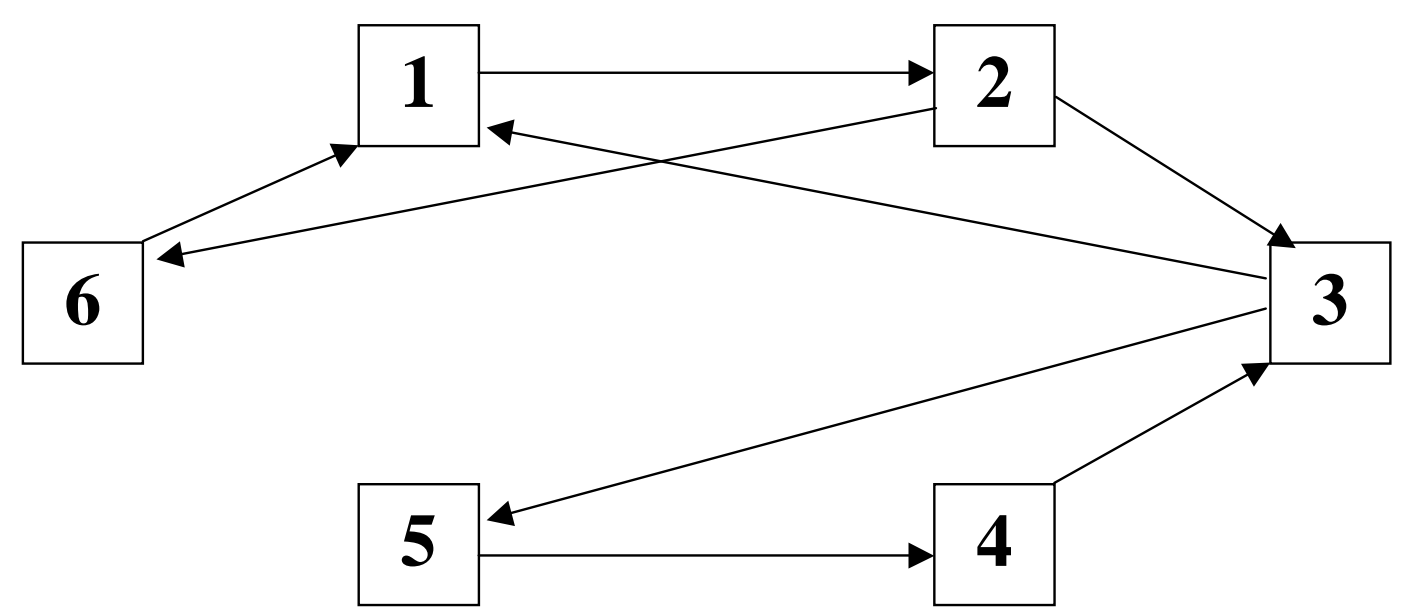

Figure 3: A Weak Nash Configuration with 8 links 


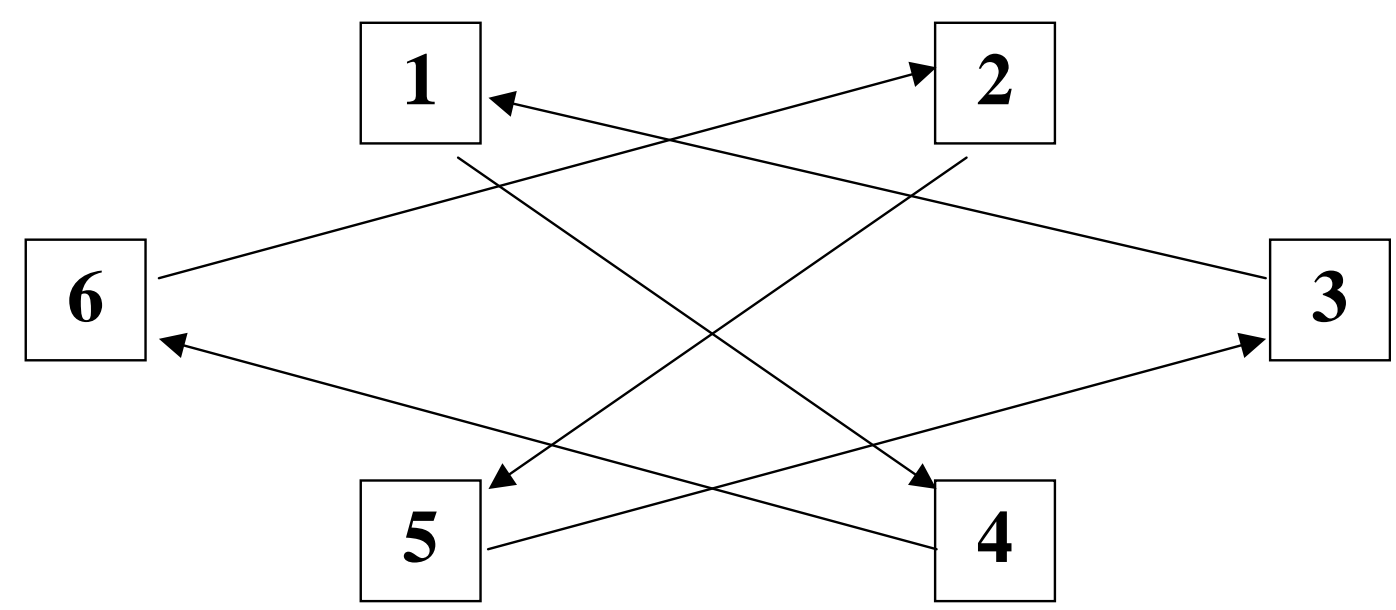

Figure 4: An Efficient Non-Focal wheel (parameter set \#3)

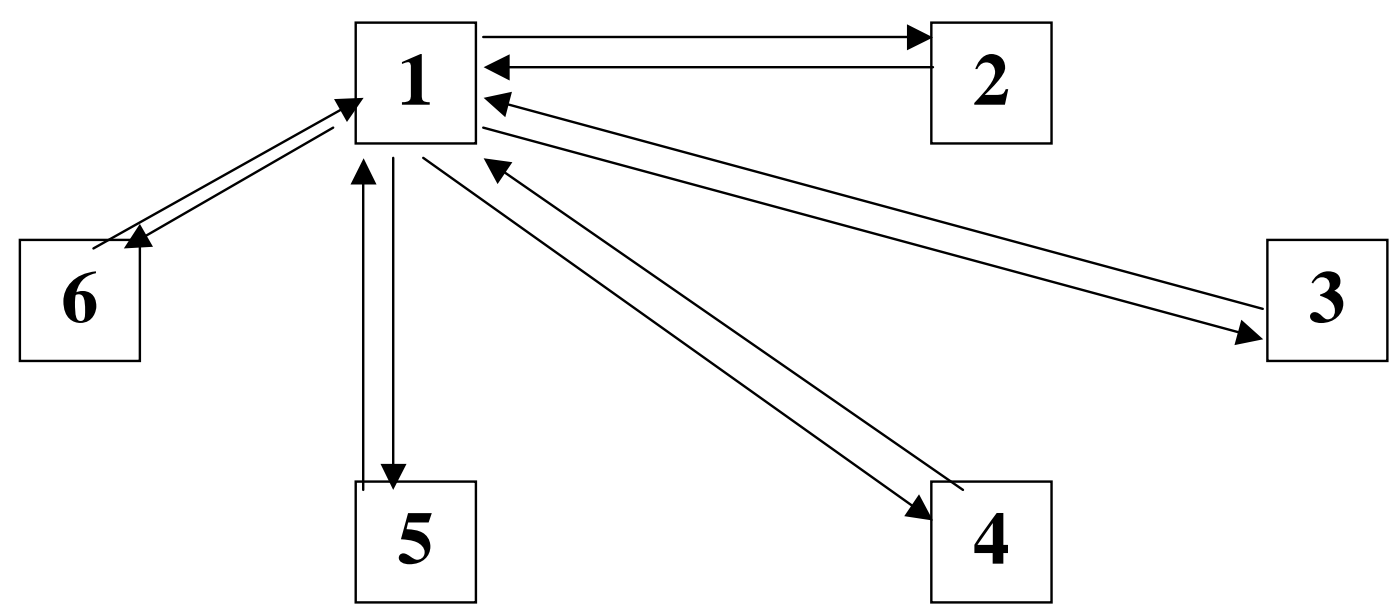

Figure 5: An Efficient Star (parameter set \#4)

\begin{tabular}{|ll|}
\hline Property & Value of Property \\
- number of agents & 6 \\
- flow quality & no decay \\
- flow direction & one way \\
- actors & individuals at nodes \\
\hline
\end{tabular}

Table 3: Experimental Design: Common

Features Series One and Series Two 


\begin{tabular}{|ll|}
\hline Series $\mathbf{1}$ & Series $\mathbf{2}$ \\
$\cdot 5$ experiments & $\cdot 7$ experiments \\
$\cdot$ conducted manually & $\cdot$ conducted over computer \\
$\cdot$ simultaneous moves & $\cdot$ continuous opportunity to move \\
$\cdot$ no cost of adjustment & $\cdot$ adjustment cost imposed \\
$\cdot$ random stopping between $10-20$ rounds & $\cdot$ random stopping between $15-20$ rounds \\
\hline
\end{tabular}

Table 4: Experimental Design: Specific Features Series One and Series Two

\begin{tabular}{|c|c|c|c|c|c|}
\hline \multicolumn{6}{|c|}{ Stop if dice roll $\geq$} \\
\hline Round & Rule 1 & Rule 2 & Round & Rule 1 & Rule 2 \\
\hline 10 & 12 & - & 15 & 7 & 12 \\
\hline 11 & 11 & - & 16 & 6 & 10 \\
\hline 12 & 10 & - & 17 & 5 & 8 \\
\hline 13 & 9 & - & 18 & 4 & 6 \\
\hline 14 & 8 & - & 19 & 3 & 4 \\
\hline 15 & 7 & 12 & 20 & 2 & 2 \\
\hline
\end{tabular}

Table 5: Stopping Rules

\begin{tabular}{|llllll|}
\hline & Series 1 & & & Series 2 & \\
981106 & Parameters & Rounds & Experiment & Parameters & Rounds \\
990115 & Set 1 & $1-10$ & 010528 & Set 2 & $1-19$ \\
990128 & Set 1 & $1-15$ & $010607 \mathrm{a}$ & Set 2 & $1-17$ \\
$990212 \mathrm{a}$ & Set 1 & $1-16$ & $010607 \mathrm{~b}$ & Set 2 & $1-18$ \\
$990212 \mathrm{~b}$ & Set 1 & $1-13$ & $010613 \mathrm{a}$ & Set 2 & $1-7$ \\
& Set 1 & $1-13$ & & Set 3 & $8-12$ \\
& & & & Set 4 & $13-16$ \\
& & & $010613 \mathrm{~b}$ & Set 2 & $1-9$ \\
& & & & Set 3 & $10-16$ \\
& & & $010614 \mathrm{a}$ & Set 2 & $1-6$ \\
& & & $010614 \mathrm{~b}$ & Set 2 & $7-17$ \\
& & & & & $1-17$ \\
\hline
\end{tabular}

Table 6: Experimental Design: Parameters 


\begin{tabular}{|c|c|c|}
\hline Series 1 & & \\
\hline Experiment & Rounds & Result \\
\hline 981106 & 10 & No convergence \\
\hline 990115 & 15 & No convergence \\
\hline 990128 & 16 & No convergence \\
\hline $990212 \mathrm{a}$ & 13 & Converged to focal wheel in rounds $9-13$ \\
\hline $990212 \mathrm{~b}$ & 13 & Converged to focal wheel in rounds $11-13$ \\
\hline Series 2 & & \\
\hline 010528 & 19 & Converged to non-focal wheel in rounds $17-19$ \\
\hline 010607a & 17 & No convergence \\
\hline 010607b & 18 & Converged to inefficient weak Nash in rounds $16-18$ \\
\hline 010613a & 16 & $\begin{array}{l}\text { Converged to focal wheel in rounds } 5-7 \\
\text { Converged to efficient non-focal wheel in rounds } 10-12 \\
\text { No convergence in rounds } 13-16\end{array}$ \\
\hline 010613b & 16 & $\begin{array}{l}\text { Converged to focal wheel in rounds } 7-9 \\
\text { Converged to efficient non-focal wheel in rounds } 14-16\end{array}$ \\
\hline 010614a & 17 & $\begin{array}{l}\text { Converged to focal wheel in rounds } 4-6 \\
\text { Converged to efficient non-focal wheel in rounds } 15-17\end{array}$ \\
\hline 010614b & 17 & Converged to focal wheel in rounds $15-17$ \\
\hline
\end{tabular}

Table 7: Summary Data: All Experiments

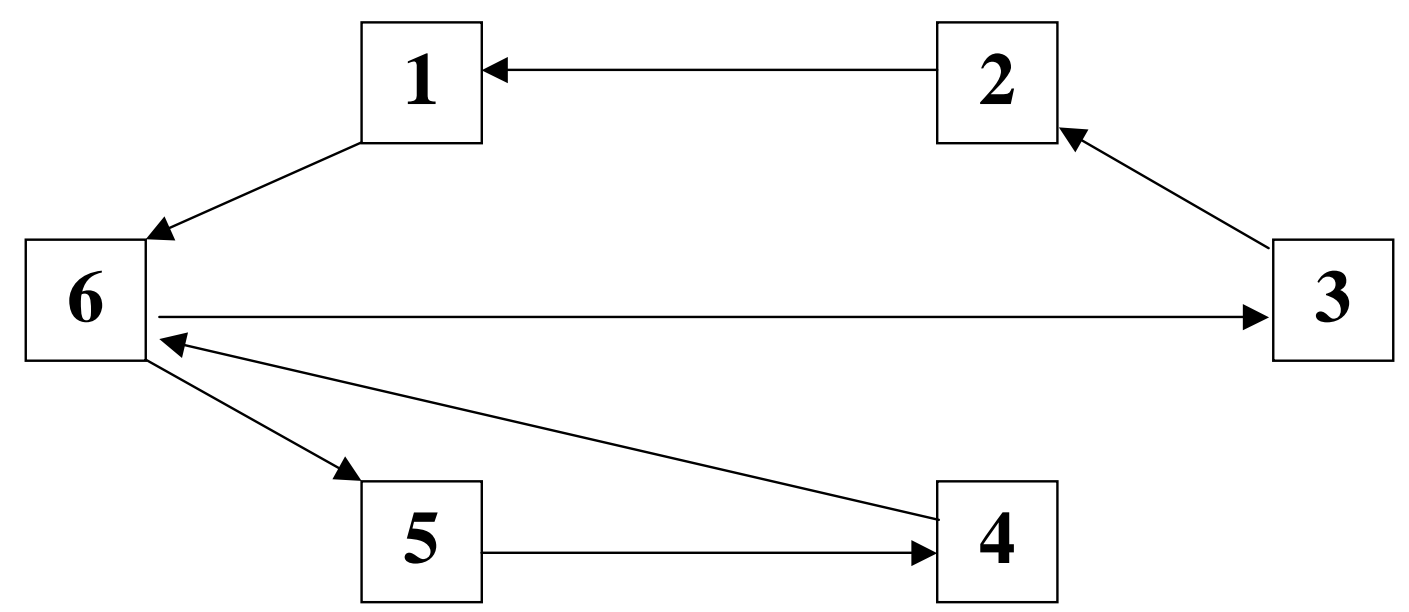

Figure 6: Stationary Weak Nash Configuration in Experiment 010607b 

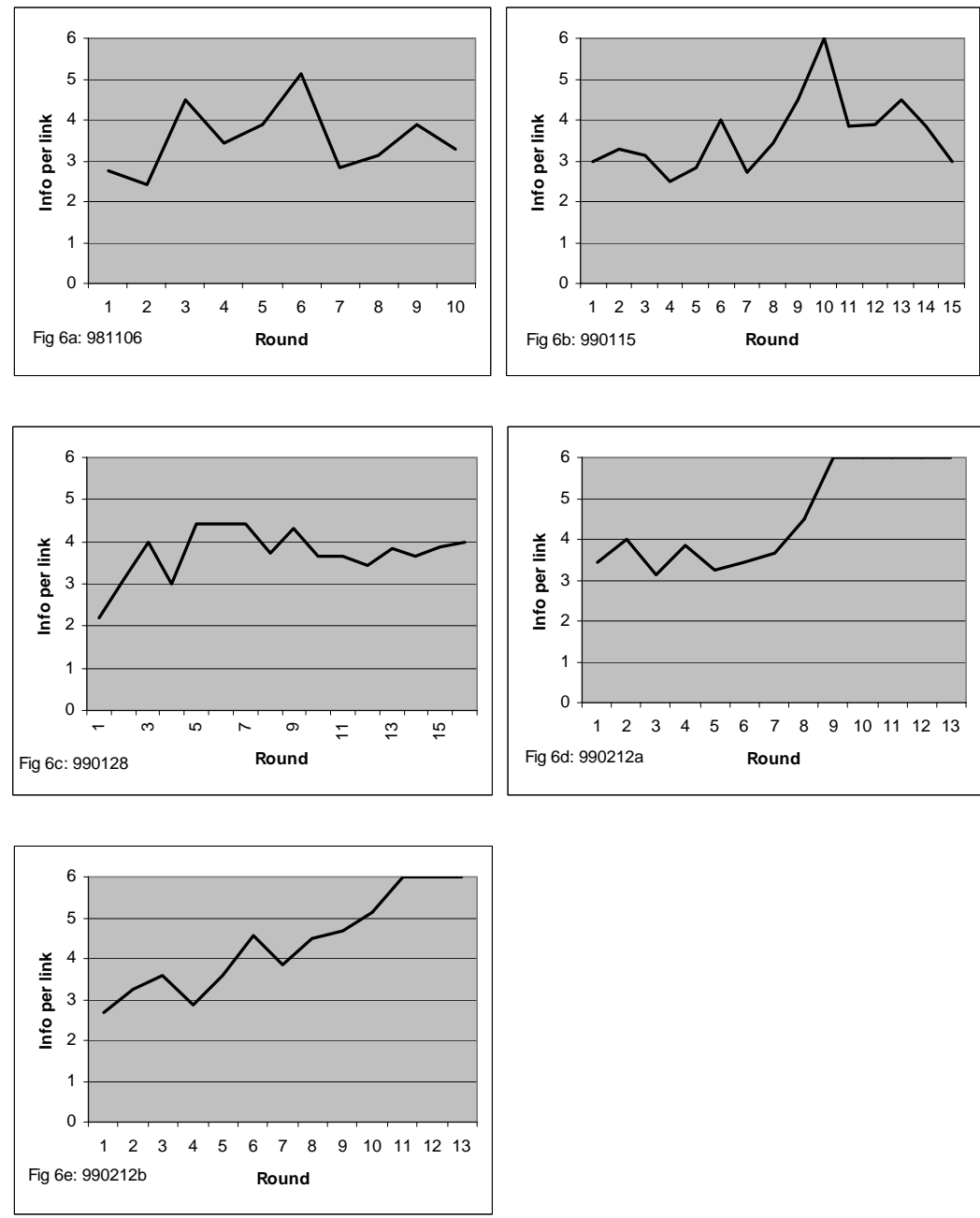

Figure 7: Series One Network Efficiency 

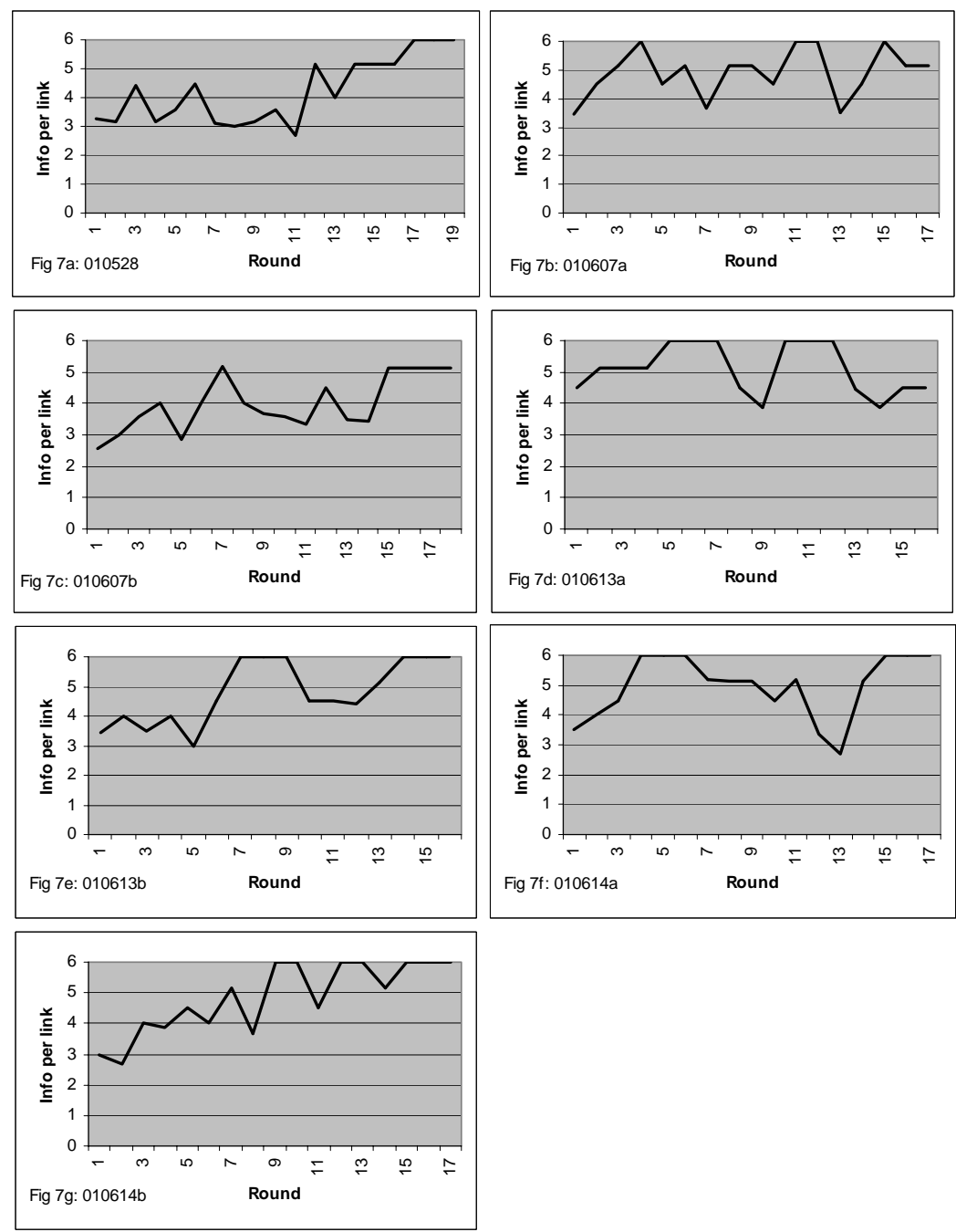

Figure 8: Series Two Network Efficiency 


\begin{tabular}{|c|c|c|c|c|c|c|c|c|}
\hline & \multicolumn{10}{|c|}{ Error Level } \\
\cline { 2 - 9 } & \multicolumn{3}{|c|}{ All 30 Agents } & \multicolumn{1}{|c|}{12} & Agents using Best Response \\
\hline Level of Significance & $1 \%$ & $5 \%$ & $10 \%$ & $25 \%$ & $1 \%$ & $5 \%$ & $10 \%$ & $25 \%$ \\
\hline $1 \%$ & 0 & 0 & 1 & 14 & 0 & 0 & 1 & 5 \\
\hline $2 \%$ & 0 & 0 & 1 & 12 & 0 & 0 & 1 & 4 \\
\hline $5 \%$ & 0 & 0 & 0 & 8 & 0 & 0 & 0 & 1 \\
\hline $10 \%$ & 0 & 0 & 0 & 2 & 0 & 0 & 0 & 1 \\
\hline
\end{tabular}

Table 8: Number of Agents for Whom the Best Response

Decision Rule Cannot be Rejected

\begin{tabular}{|c|c|c|c|c|c|}
\hline & \multicolumn{5}{|c|}{ Error Level } \\
\hline Level of Significance & $1 \%$ & $2 \%$ & $5 \%$ & $10 \%$ & $25 \%$ \\
\hline $1 \%$ & 6 & 7 & 8 & 12 & 20 \\
\hline $2 \%$ & 6 & 6 & 8 & 12 & 18 \\
\hline $5 \%$ & 6 & 6 & 7 & 10 & 16 \\
\hline $10 \%$ & 6 & 6 & 6 & 10 & 15 \\
\hline
\end{tabular}

Table 9: Number of Agents for Whom Simply Strategic Behavior Cannot be Rejected

\begin{tabular}{|c|c|c|c|c|c|c|c|c|}
\hline \multirow{2}{*}{} & \multicolumn{7}{|c|}{ Error Level } \\
\cline { 2 - 9 } & \multicolumn{7}{|c|}{ All 30 Agents } & \multicolumn{1}{c|}{$17 \frac{1}{2}$ Agents using SScc } \\
\hline Level of Significance & $1 \%$ & $5 \%$ & $10 \%$ & $25 \%$ & $1 \%$ & $5 \%$ & $10 \%$ & $25 \%$ \\
\hline $1 \%$ & 3 & 5 & 10 & 17 & 3 & 5 & 10 & 14 \\
\hline $2 \%$ & 3 & 5 & 9 & 15 & 3 & 5 & 9 & 12 \\
\hline $5 \%$ & 3 & 5 & 5 & 13 & 3 & 5 & 5 & 12 \\
\hline $10 \%$ & 3 & 5 & 5 & 11 & 3 & 5 & 5 & 11 \\
\hline
\end{tabular}

Table 10: Number of Agents for Whom the Counter-Clockwise Simple Strategic Behavior (SScc) Cannot be Rejected 


\begin{tabular}{|c|c|c|c|c|c|}
\hline & \multicolumn{5}{|c|}{ Experiment $^{28}$} \\
\hline Level of Significance & 981106 & 990115 & 990128 & $990212 \mathrm{a}$ & $990212 \mathrm{~b}$ \\
\hline $1 \%$ & 4 & 4 & 3 & 6 & 6 \\
\hline $2 \%$ & 4 & 5 & 4 & 6 & 6 \\
\hline $5 \%$ & 4 & 5 & 4 & 6 & 6 \\
\hline $10 \%$ & 4 & 6 & 4 & 6 & 6 \\
\hline
\end{tabular}

Table 11: Agents For Whom Randomness is Rejected in Favor of Counter-Clockwise Simple Strategic Behavior (SScc)

\begin{tabular}{|c|c|c|}
\hline & \multicolumn{2}{|c|}{ Experiment } \\
\hline Level of Significance & $990212 \mathrm{a}$ & $990212 \mathrm{~b}$ \\
\hline $1 \%$ & 4 & 4 \\
\hline $2 \%$ & 4 & 4 \\
\hline $5 \%$ & 4 & 5 \\
\hline $10 \%$ & 4 & 5 \\
\hline
\end{tabular}

Table 12: Agents for Whom Randomness is Rejected in Favor of Counter-Clockwise Simple Strategic Behavior Before Convergence Occurs

\begin{tabular}{|c|c|c|c|c|c|}
\hline & \multicolumn{5}{|c|}{ Experiment } \\
\hline Before Convergence: & 981106 & 990115 & 990128 & $990212 \mathrm{a}$ & $990212 \mathrm{~b}$ \\
\hline Observations of ssa $(\gamma)$ & 23 & 41 & 21 & 25 & 29 \\
\hline Rounds $(n)$ & 10 & 15 & 16 & 8 & 10 \\
\hline Rate of ssa $\left(\frac{\gamma}{n}\right)$ & 2.3 & 2.733 & 1.313 & 3.125 & 2.9 \\
\hline Converged $(y)$ & No & No & No & Yes & Yes \\
\hline
\end{tabular}

Table 13: Observations of Counter-Clockwise Simple Strategic Behavior

\footnotetext{
${ }^{28}$ The hypothesis tested here is that agents choose among all strategies (including SScc) randomly. The results, therefore, reject the hypothesis for a majority of agents that a random selection generated the observed sample of frequent SScc selection. Thus, this leads to the conclusion that agents chose according to the SScc strategy with greater than random probability.
} 


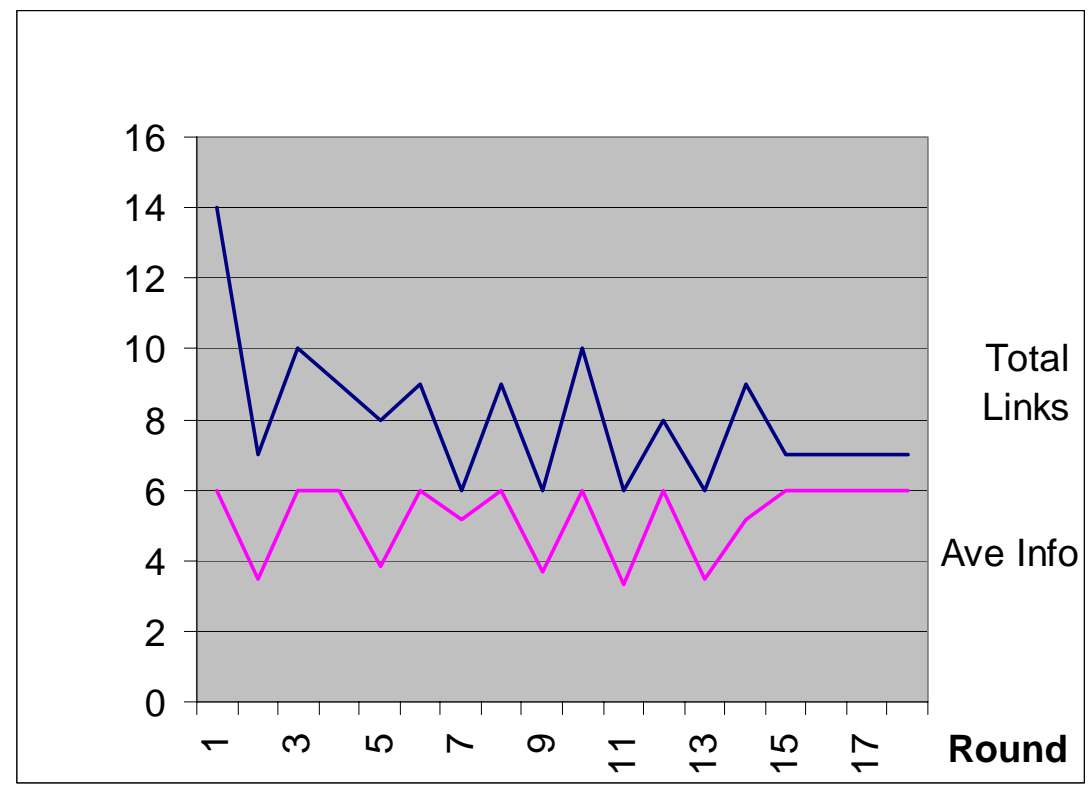

Figure 9: Total Links and Average Information Received

\begin{tabular}{|c|c|c|}
\hline Variable & Estimate & t-statistic \\
\hline$\alpha$ & 4.86 & 7.66 \\
\hline$\beta$ & -1.00 & -8.29 \\
\hline$R^{2}=0.32$ & $n=148$ & \\
\hline
\end{tabular}

Table 14: "Aggregate Level Quasi-Best Responsiveness" 